\title{
Theta band behavioral fluctuations synchronized interpersonally during cooperation
}

\author{
Cheng Wang ${ }^{1}\left(\mathbb{D} \cdot{\text { Hong } \mathrm{Li}^{1} \cdot \text { Lei Jia }}^{1} \cdot\right.$ Feiming $\mathrm{Li}^{1} \cdot$ Jun Wang ${ }^{1}$
}

Published online: 12 February 2020

(C) The Psychonomic Society, Inc. 2020

\begin{abstract}
Human behavior fluctuates. A growing body of evidence has demonstrated that behavioral performance in perception fluctuates rhythmically, with dynamics closely resembling spectral features of neural oscillations. However, it is unclear whether the behavioral fluctuations in a complex cooperation context can also express similar rhythmic features, and, more importantly, whether these behavioral rhythms are synchronized among co-actors in a neurophysiologically relevant manner. To answer these questions, we applied a time-resolved approach, previously used for probing individual-level behavioral oscillations in perception, in a complex social interaction context, and further probed dyad-level behavioral synchrony. Twenty pairs of male participants completed, in dyad, joint-action tasks with cooperation or competition demand. We extracted behavioral rhythms from ongoing cooperative performance and measured behavioral synchrony by computing the phase coherence of these behavioral rhythms between dyad members. Despite the absence of significant behavioral oscillations in individuals' amplitude spectrum, we observed enhanced theta-band phase coherence between co-actors' behavioral rhythms during cooperation compared to competition conditions. These results indicate that cooperative behaviors of co-actors fluctuated synchronously within the theta band, providing a behavioral counterpart of theta-band interbrain synchrony in cooperation reported in previous hyperscanning studies. Furthermore, the observed behavioral synchrony could be used as a sensitive predictor of cooperation pattern, as evidenced by its significant correlation with leader-follower relationship during cooperation.
\end{abstract}

Keywords Cooperation $\cdot$ Interpersonal behavioral synchrony $\cdot$ Behavioral oscillation $\cdot$ Theta-band coherence $\cdot$ Joint-action task

\section{Introduction}

Human behavior fluctuates over time. These fluctuations are usually regarded as measurement noise (Landau \& Fries, 2012), yet recent evidence has demonstrated that they are informative of rhythmic characteristics that closely resemble the spectral features of neural oscillations (Benedetto \& Morrone, 2017; Fiebelkorn \& Kastner, 2019; Fiebelkorn, Saalmann, \& Kastner, 2013; Ho, Burr, Alais, \& Morrone, 2019; Ho, Leung, Burr, Alais, \& Morrone, 2017; Huang, Chen, \& Luo, 2015; Landau \& Fries, 2012; Re, Inbar,

Electronic supplementary material The online version of this article (https://doi.org/10.3758/s13423-020-01711-0) contains supplementary material, which is available to authorized users.

Jun Wang

jun.wang@zjnu.edu.cn

1 Department of Psychology, Zhejiang Normal University, \#688 Yingbin Rd, Jinhua 321001, Zhejiang Province, China
Richter, \& Landau, 2019; Song, Meng, Chen, Zhou, \& Luo, 2014; Tomassini, Spinelli, Jacono, Sandini, \& Morrone, 2015). However, as existing evidence is restricted within the study of perception, it is unclear whether complex cooperative behaviors also fluctuate rhythmically and, more importantly, whether these fluctuations are synchronized among co-actors in a neurophysiologically relevant manner (e.g., phase coherence).

Cooperation is of vital importance to human society, on which human beings rely to survive and thrive. One important form of cooperation is synchronized behaviors. For many cooperative activities, synchronized behavior is crucial for optimal performance. In constructing a pyramid in ancient Egypt, for example, hundreds of slaves needed to synchronize their pulling or lifting to move the massive stone blocks. The synchronization of co-actors' behavior has been investigated in kinematic studies using body movement dynamics recorded via motion-tracking devices (Chauvigné, Walton, Richardson, \& Brown, 2019; Cuijpers, Den Hartigh, Zaal, \& de Poel, 2019; Richardson, Garcia, Frank, Gregor, \& Marsh, 2012; 
Silva et al., 2016). However, these studies focused mainly on the synchronization of overt movements, without concerning the spectral feature or the underlying inner cognitive and neurophysiological processes. And the movement dynamics they studied are too slow (frequencies $<1 \mathrm{~Hz}$, e.g., $0.6 \mathrm{~Hz}$ for chair rocking (Richardson et al., 2012), or $\sim 30-50$ strokes per minute for rowing (Cuijpers et al., 2019)) to inhabit timescales of change as fast as the timescales of cognitive and neurophysiological dynamics.

The present study aimed to probe the spectral synchrony of behavioral fluctuation during cooperation with a timescale as short as $\sim 50 \mathrm{~ms}$ (frequencies as high as $\sim 20$ $\mathrm{Hz}$ ) using a time-resolved approach. This approach has been successfully used to track spectral features (4-12 $\mathrm{Hz}$ ) in the fluctuations of behavioral performance (accuracy, reaction times, sensitivity, or decision criteria) of visual (Landau \& Fries, 2012; Tomassini et al., 2015; VanRullen, 2016) and auditory (Ho et al., 2019; Ho et al., 2017) perception. Using this approach in a wellestablished cooperation paradigm (Cheng, Li, \& Hu, 2015; Cui, Bryant, \& Reiss, 2012), we were able to access the fine-grained time course of behavioral fluctuation in cooperation, from which band-specific behavioral rhythms can be extracted and phase coherence between co-actors' behavioral rhythms can be computed. Thetaband interbrain phase coherence has been reported to be enhanced during social cooperation (Hu et al., 2018; Kawasaki, Yamada, Ushiku, Miyauchi, \& Yamaguchi, 2013; Liu et al., 2018; Sänger, Müller, \& Lindenberger, 2012). Additionally, theta-band rhythm has been implicated in prediction (Huang et al., 2015), cognitive control (Cavanagh \& Frank, 2014), and behavioral adaptation (Billeke, Zamorano, Cosmelli, \& Aboitiz, 2012; Cavanagh, Frank, Klein, \& Allen, 2010), all of which are essential for cooperation. Taken together, we expect the co-actors' behavioral fluctuations to be synchronized within the theta band.

\section{Methods}

\section{Participants}

Twenty pairs of male college students (all right-handed, average age $20.72 \pm 1.45$ years) with normal or corrected-to-normal vision participated in the experiment. Two extra pairs of participants were eliminated from the study due to an inadequate number of trials after data trimming $>30 \%$ of trials were discarded). This study recruited only males because a recent study reported that male-male dyads exhibited better cooperative performance than male-female and female-female dyads (Cheng et al., 2015). Participants were randomly paired, and members of pairs were not acquainted with each other before the experiment. The participants provided written informed consent before the experiment and received monetary compensation depending on their performance. This study conforms to the guidelines of the Declaration of Helsinki and was approved by the research ethics committee of Zhejiang Normal University.

\section{Stimuli and procedure}

Using a time-resolved approach, we recruited 20 pairs of adults in a well-established cooperation paradigm (Cui et al., 2012). Each pair of participants sat side-by-side in front of a monitor (Fig. 1b), with the participant on the left side denoted as participant \#1 and the one on the right as participant \#2. They were asked to complete a cooperation task and a competition task (as a control condition) separately in 2 days. In both tasks, each pair of participants were informed that a green disk ("go" signal) would appear after a gray ring ("ready" signal) and they are required to press a button after seeing the green disk (Fig. 1a). Reaction times (RTs) were collected. In the cooperation task, participants were asked to minimize their RT difference. If the difference was smaller than a threshold (defined as one-eighth of the sum of their RTs in the current trial; Cui et al., 2012), both participants earned one point; otherwise, they both lost one point. In the competition task, participants were asked to press keys as fast as possible upon seeing the green signal; the participant with the faster response won a point and the other lost one point. Participants were asked to maximize their points, and their monetary compensates will depend on their winning scores in each task. After both participants responded, feedbacks were presented with information to reinforce cooperation or competition in respective tasks. It is worth noting that stimuli were exactly the same for cooperation and competition tasks before feedback screen, and participants needed to adjust their responses according to different task demands (see Supplementary Material for details)

Critically, to achieve fine-grained temporal assessment of behavior performance, we used a time-resolved approach such that the stimulus onset asynchronies (SOAs) between the "ready" and "go" signals were arranged from $0.2 \mathrm{~s}$ to $1 \mathrm{~s}$ in steps of $20 \mathrm{~ms}$, corresponding to a sampling frequency of 50 $\mathrm{Hz}$. The assumption is that the "ready" signal serves as a time reference and a salient event to reset the phase of ongoing behavioral rhythms (VanRullen, 2016), leading to reproducible cooperative and competitive dynamics over multiple trials (Fig. 1c). An exhaustive set of SOAs allows densely sampling the rapid fluctuation of ongoing behavioral performance, such that a fine-grained time course for each condition can be generated for each participant (Figs. 2a and 2b). The resulting time courses were subsequently analyzed for oscillation and interpersonal synchronization. 
a

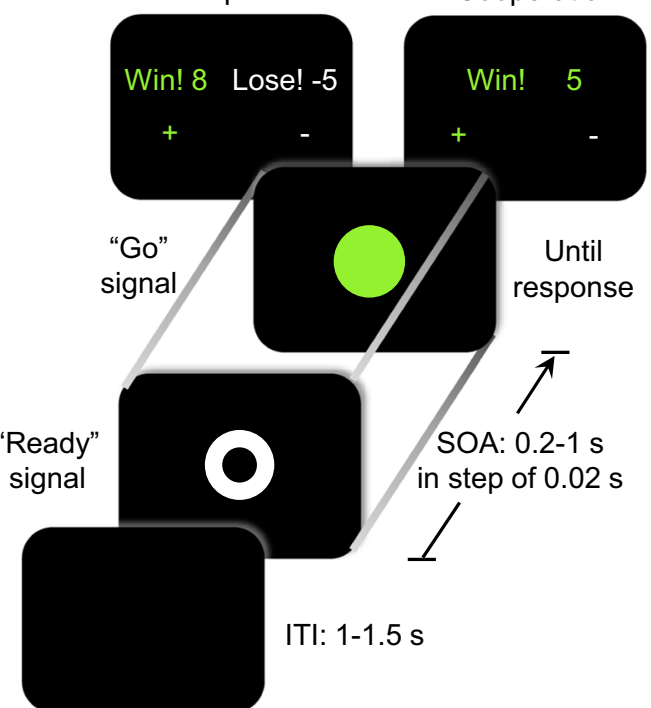

b

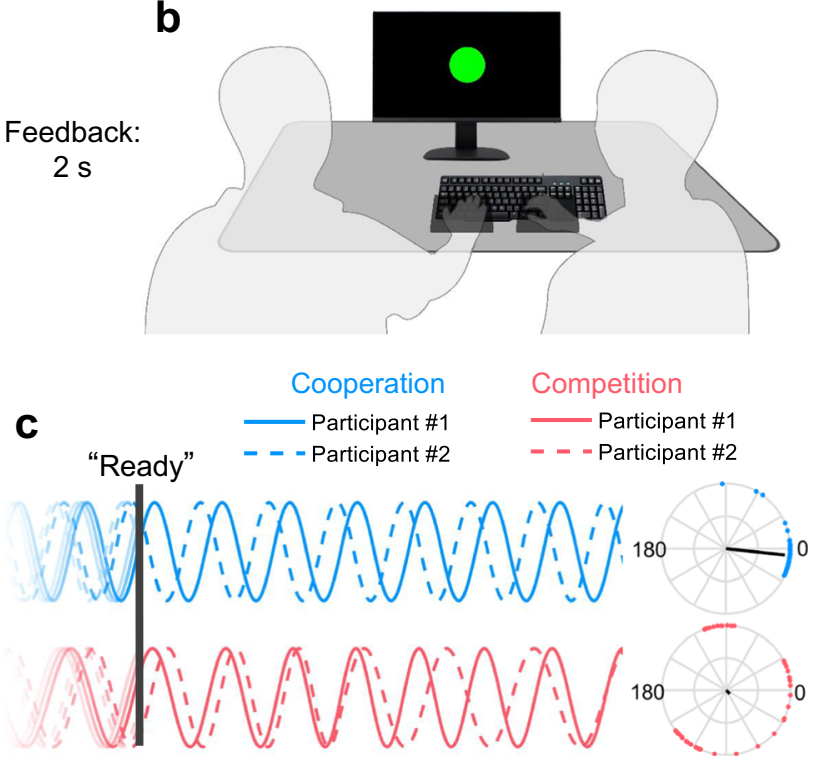

fluctuations of participant \#1 and \#2 in the cooperation (blue) vs. the competition (red) conditions. For cooperation, the phase differences between the two co-actors are consistent across time, as indicated by the relatively more clustered blue dots in the polar system, representing a high interpersonal phase coherence. For competition, the phase differences were more inconsistent across time, as indicated by the relatively more evenly distributed red dots in the polar space, representing a low interpersonal phase coherence. The length of the black lines in the polar space represents the values of interpersonal phase coherence

\section{Results}

Figures $2 \mathrm{a}$ and $2 \mathrm{~b}$ illustrate the raw RT time courses averaged over all dyads for participant \#1 (blue) and participant \#2 (red) as a function of SOA in the cooperation (A) and competition (B) conditions. Generally, the RT traces decreased over time courses, demonstrating a second-order polynomial trend. To

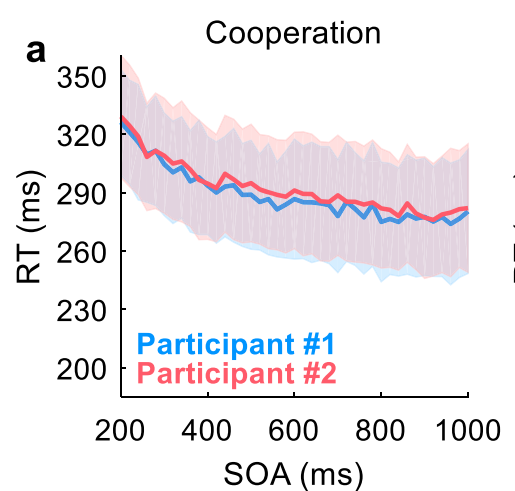

Fig. 2 Grand average reaction time (RT) time course. (a and b) Grand average RT time courses for the cooperation (a) and competition (b) conditions. Blue and red lines represent participant \#1 and participant $\# 2$, respectively; shaded regions denote \pm SEM. Time courses were averaged separately for "participant \#1" and "participant \#2" for display purposes. Note that the grand averaged data only loosely represent the fluctuating characteristic because the main findings were generated from single-participant spectral or single-dyad coherence analysis. (c) Grand
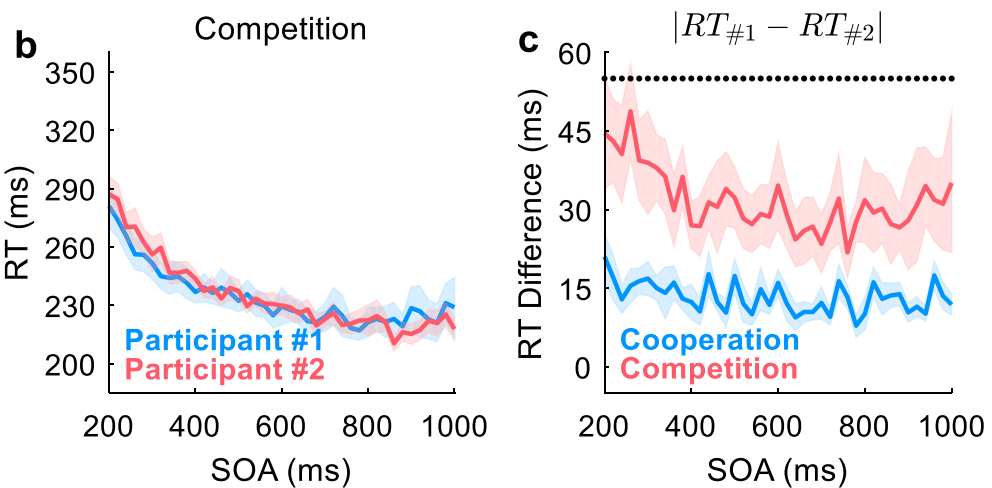

average time courses of absolute values of RT differences between participant \#1 and participant \#2. In the title, "RT \#1" denotes RT for participant \#1, and " $\mathrm{RT}_{\# 2}$ " denotes RT for participant \#2. Blue and red lines represent absolute RT differences in the cooperation and competition conditions, respectively; shaded regions denote \pm SEM. Black dots indicate SOAs in which the difference between the cooperation and competition conditions was statistically significant (ps $<0.05$, FDR corrected) 
better characterize the fluctuating pattern in the time courses, we removed the trend using robust detrending (de Cheveigné \& Arzounian, 2018) (see Fig. S2 for fluctuations of the time courses of each dyad after normalizing and detrending). Moreover, Fig. 2c shows the time courses of absolute values of RT differences between participant \#1 and participant \#2 as a function of SOA. The absolute values of RT differences were significantly smaller in the cooperation than the competition conditions (permutation test, ps $<0.05$, p-values were corrected by a false discovery rate (FDR) of 0.1 (Genovese, Lazar, \& Nichols, 2002)). This finding was consistent with our experimental setup that participants were required to minimize their RT difference in the cooperation task but to respond as fast as possible in the competition task.

To measure the characteristics of oscillatory components in the ongoing behavioral performance, raw RT time courses of each participant in each condition were normalized, detrended, and then subjected to a fast Fourier transform. Figures $3 \mathrm{a}$ and $3 \mathrm{~b}$ show the grand average amplitude spectrum averaged across all participants (both participants in all dyads) in the cooperation and competition conditions, respectively. To determine whether the spectrums simply reflected a random fluctuations in time, we performed permutation tests to examine statistical significance. The dashed lines in Figs. 3a and $3 \mathrm{~b}$ represent the cutoff for statistical significance (ps < 0.05 , FDR corrected). As shown in the figures, the amplitude spectrums in both conditions revealed no significant peak. Additionally, the spectral amplitude was generally higher in the cooperation than the competition conditions. However, the effect was significant only for several frequencies (as indicated by black dots in Fig. 3c; permutation test, ps < 0.05 , FDR corrected) due to larger fluctuations in cooperation than competition (as shown in Figs. 2a and 2b).

Next, to examine the synchronization between the two RT time courses of participant \#1 and participant \#2 in a dyad, we computed phase coherence between the two participants in each dyad. Figures $3 \mathrm{~d}$ and $3 \mathrm{e}$ illustrate the coherence spectrum averaged over all dyads in the cooperation and competition conditions, respectively. To assess whether the coherence was due to a random relationship between the two time courses in a dyad, we performed permutation tests to examine statistical significance. The dashed lines in Figs. $3 \mathrm{~d}$ and $3 \mathrm{e}$ represent the cutoff for statistical significance ( $\mathrm{ps}<0.05$, FDR corrected). The results revealed significant coherences in the theta and alpha band $(\sim 4-10 \mathrm{~Hz})$ in the cooperation condition but not in the competition condition. However, only theta-band
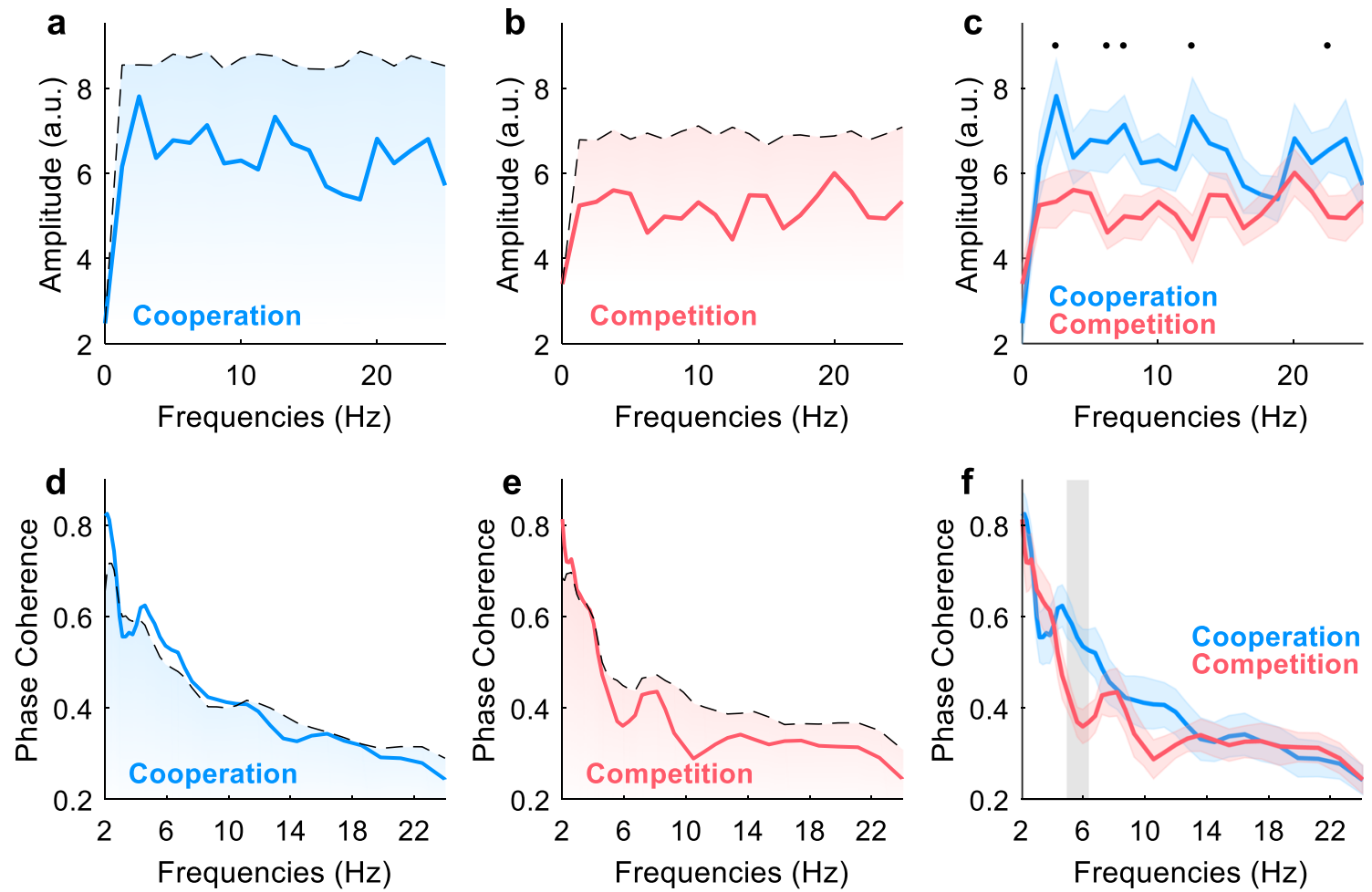

Fig. 3 Grand average amplitude and coherence spectrum. (a and b) Grand average amplitude spectrum for the cooperation (a) and competition (b) conditions. Dashed lines represent the cutoff of statistical significance ( $\mathrm{ps}<0.05$, FDR corrected). (c) Comparison of the amplitude spectrum between the cooperation (blue) and competition (red) conditions. Shaded regions denote \pm SEM. Black dots indicate the frequencies for which the difference between cooperation and competition was statistically significant (permutation test, ps $<0.05$,
FDR corrected). (d and e) Grand average coherence spectrum for the cooperation (d) and competition (e) conditions. Dashed lines represent the cutoff of statistical significance ( $\mathrm{ps}<0.05$, FDR corrected). (f) Comparison of the coherence spectrum between the cooperation (blue) and competition (red) conditions. Shaded blue and red regions denote \pm SEM. The gray-shaded rectangle indicates the frequencies for which the difference between cooperation and competition was statistically significant (permutation test, $\mathrm{ps}<0.05$, FDR corrected) 
coherence $(\sim 5-6 \mathrm{~Hz})$ exhibited a significant difference between the cooperation and competition conditions, with enhanced coherence observed for the cooperation condition (permutation test, ps $<0.05$, FDR corrected) (and see Fig. S3 for coherence difference in each dyad).

Recently, several studies have noted that cooperation is improved when leader-follower relationship is defined (Fairhurst, Janata, \& Keller, 2014; Jiang et al., 2015; Konvalinka et al., 2014). In the present study, the leader was defined as the participant who had more trials with faster responses, whereas the follower was defined as his partner. Here, to quantify the leader-follower relationship within a dyad, we coined the term "balance index," calculated as the ratio of the follower's summed counts of faster-responding trials to the leader's summed counts of faster-responding trials (see Fig. 4a for an exemplary dyad). This index ranges from 0 to 1 , with zero indicating that the leader responded faster in all trials (thus forming a fully unbalanced relationship) and one indicating the absence of a leader/ follower in a dyad (thus forming a fully balanced relationship). Indeed, in accordance with previous findings (Fairhurst et al., 2014; Konvalinka et al., 2014), we found a significant negative correlation (Spearman's rho $=-0.472, p=0.037$, Fig. 4b) between this index and cooperative performance, which was quantified by the winning score (points won minus points lost) in the cooperation task.

Finally, to examine whether theta-band coherence in the cooperation task was of behavioral relevance, we calculated the correlation of theta-band coherence with balance index and winning score. Theta-band coherence was estimated by the average coherence across \pm 3 frequency points around the peak of the single-dyad's coherence spectrum in the range of $3-8 \mathrm{~Hz}$ in the cooperation task. We found that theta-band coherence was significantly negatively correlated with balance index (Spearman's rho $=-0.537, p=0.016$, Fig. 4c).
Additionally, theta-band coherence was not significantly correlated winning score (Spearman's $r h o=0.385, \mathrm{p}=0.095$ ). This might suggest winning score is not an appropriate indicator for cooperation compared to balance index, since the criterion we used to quantify winning or losing was, in some degree, arbitrary. By contrast, alpha-band $(8-12 \mathrm{~Hz})$ coherence in cooperation did not significantly correlate with winning score (Spearman's rho $=-0.226, \mathrm{p}=0.337$ ) or balance index (Spearman's $r h o=0.015, \mathrm{p}=0.952$ ).

\section{Discussion}

The present study reports, for the first time, that cooperative behaviors of co-actors fluctuated synchronously, in a manner that resembles the spectral features of interbrain synchrony found in hyperscanning studies (Hu et al., 2018; Kawasaki et al., 2013; Liu et al., 2018; Sänger et al., 2012). Using a time-resolved approach, we observed that theta-band interpersonal behavioral coherence was enhanced during cooperation relative to competition and significantly correlated with leader-follower relationships during cooperation, pinpointing a unique role of thetaband interpersonal behavioral coherence during cooperation.

Using a time-resolved approach, previous studies have repeatedly reported behavioral oscillation in visual (Landau \& Fries, 2012; Tomassini et al., 2015) and auditory (Ho et al., 2019; Ho et al., 2017) detection task, implying that perception and cognition operate periodically, as a succession of cycles mirroring the underlying neural oscillations (Landau, 2018; VanRullen, 2013, 2016). Here, we used this approach in a complex social interaction context to probe behavioral oscillation in cooperation and competition. However, we did not find significant behavioral oscillation in individual's amplitude spectrum. This might be due to task difference. The task a
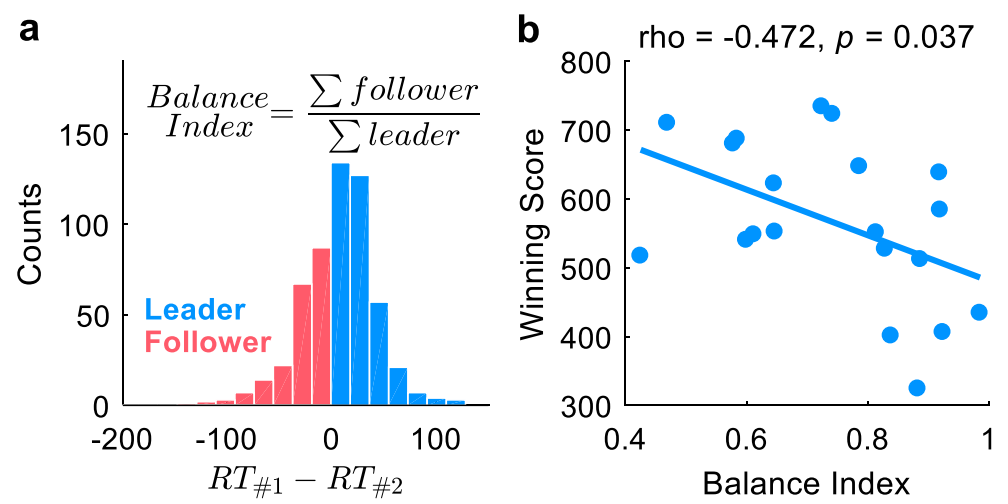

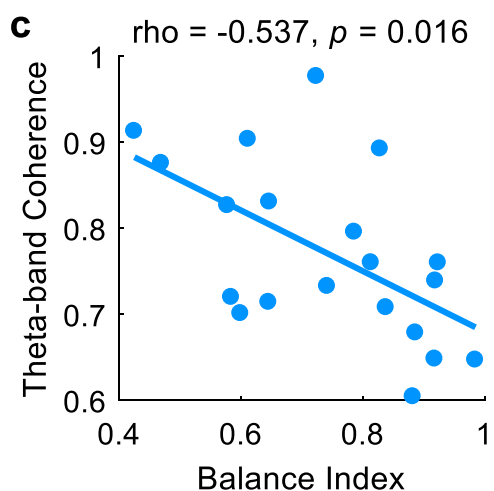

Fig. 4 Correlations between phase coherence, balance index, and winning score. (a) Calculation of balance index in the cooperation task. The plot shows the histogram of trial counts as a function of reaction time (RT) difference (participant \#1 - participant \#2) in an exemplary dyad. First, we defined the participant in a dyad who had more trials with faster response as the leader (blue) and the other participant as the follower (red). Second, balance index was calculated as the ratio of the follower's summed counts of faster-responding trials to the leader's summed counts of faster-responding trials. A higher balance index indicates a less clear leader/follower roles in a dyad. (b) Balance index was significantly correlated with winning score in the cooperation condition ( $r$ ho $=-0.472, p=0.037$ ). The line represents the best linear fit. (c) Phase coherence was significantly correlated with balance index in the cooperation condition ( $r h o=-0.537, p=0.016$ ). The line represents the best linear fit 
used in the present study was not a pure visual perception task, because participants were asked to adjust the responses to visual stimuli according to cooperation or competition demands. And these adjustments would derail the individual's behavioral responses from original behavioral rhythms, causing behavioral oscillation unable to survive statistical test.

More importantly, we further computed phase coherence between two co-actors' ongoing behavioral fluctuations to probe interpersonal behavioral synchrony, and the results revealed significant theta and alpha band interpersonal phase coherence in cooperation, in contrast to non-significant coherence in competition. The findings of theta and alpha band behavioral synchrony are consistent with EEG-based hyperscanning studies reporting larger theta and alpha band interbrain synchrony in high cooperation tasks than in low cooperation tasks ( $\mathrm{Hu}$ et al., 2018; Kawasaki et al., 2013; Liu et al., 2018). Why interpersonal phase coherence arose in cooperation? At the dyad level, co-actors' ongoing behavioral performance might have become coordinated in cooperation through top-down modulation of mutual prediction and adaptation (Konvalinka, Vuust, Roepstorff, \& Frith, 2010), leading to phase coherence in cooperation, whereas no such coordination occurred in competition. Taken together, our results, while preliminary, suggest that cooperation's effect on rhythmic dynamics can be exhibited only at the dyad level, consistent with previous observations (Cui et al., 2012) that cooperation versus competition difference was only found in dyad-level interbrain synchrony but not in individual-level spectrotemporal dynamics. This finding demonstrates, for the first time, that cooperative behaviors of co-actors fluctuated synchronously, with a dynamic resembling the spectral features of neurophysiological dynamics, providing "mirrored" evidence of interbrain synchrony (Cheng et al., 2015; Cui et al., 2012; Hu et al., 2018; Kawasaki et al., 2013; Liu et al., 2018; Sänger et al., 2012) directly in behavior dynamics. Thus, interpersonal behavioral coherence may provide a sensitive and economic tool to probe cognitive neural mechanisms of cooperation compared to costly and inconvenient brain scanning techniques.

When comparing cooperation with competition, only thetaband behavioral coherence remained significant. This suggested that theta coherence might be a unique component underling cooperation versus competition difference, while alpha coherence might be related to other cognitive components (e.g., attention) shared between tasks. The theta-band rhythms have been implicated in prediction (Huang et al., 2015), cognitive control (see Cavanagh \& Frank, 2014, for a review), and behavioral adaptation (Billeke et al., 2012; Cavanagh et al., 2010), all of which are essential for cooperation. However, alpha-band behavioral (e.g., Fiebelkorn et al., 2013; Re et al., 2019; Song et al., 2014) and neural (e.g., Bollimunta, Mo, Schroeder, \& Ding, 2011; Rohenkohl \& Nobre, 2011) oscillations have been consistently associated with attention, which could be equally engaged in both cooperation and competition. Indeed, by design, in the present study, participants need to attend to "ready" and "go" signal stimuli in both cooperation and competition tasks, leading to a comparable amount of attention involved in both conditions. These explanations are strengthened by our finding that only theta-band coherence significantly correlated with leaderfollower relationship in cooperation, while alpha-band coherence provided no such behavioral relevance.

Leader-follower relationships were used to assess the behavioral relevance of behavioral coherence because studies showed that performance was better when theta-band leader-follower roles were established (Fairhurst et al., 2014; Jiang et al., 2015; Konvalinka et al., 2014). Indeed, as shown by balance index (see Fig. 4b), in many dyads, the relationship between the two participants was not balanced regarding response speed: one participant had more trials with faster response than his partner. We assume that the more cooperative pairs of participants spontaneously adopted a leader-follower strategy when they tried to adapt to each other. Consistent with previous brain-imaging hyperscanning studies (Jiang et al., 2015; Konvalinka et al., 2014), our results showed that better cooperative performance (tagger by higher winning score) was associated with clearer leader/follower roles (tagged by lower balance index). Balance index, in turn, was negatively correlated with theta-band interpersonal behavioral coherence, indicating that dyads with a clearer leader-follower relationship tended to have higher phase coherence. In other words, theta-band fluctuations of members of a dyad tended to be better synchronized when the dyad had a clear leader. Thus, we suggest that interpersonal behavioral coherence could be used as a potential marker for leader-follower structure in cooperation, which has been suggested as a key factor impacting cooperation quality (Fairhurst et al., 2014; Jiang et al., 2015; Konvalinka et al., 2014).

In conclusion, the findings reported here provide the first evidence for interpersonal synchronization of theta-band behavioral fluctuations during cooperation and its relevance to the leader-follower relationship in cooperation. The present study shed new light on the rapidly expanding field of behavioral oscillations, by extending its application beyond perceptual behavioral rhythms revealed by individual-level amplitude spectrum to more complex social interactions revealed by dyad-level interpersonal phase coherence. Our findings pinpoint a unique role of theta-band interpersonal behavioral coherence in cooperation and provide "mirrored" evidence of interbrain synchrony directly in behavioral dynamics.

Acknowledgements This research was supported by the China National Social Science Fund in Education (2018 general project: Neural Mechanisms of Multisensory Integration Dysfunction in Autism and Related Intervention under Multi-Modality Educational Perspective. Grant No. BBA180083).

Author contributions C. Wang and J. Wang designed the experiment and wrote the paper; H. Li, L. Jia and F. Li conducted the experiment; C. Wang and J. Wang, H. Li, L. Jia and F. Li analyzed the data. All authors approved the final version of the manuscript for submission. 


\section{Compliance with ethical standards}

Competing interests The authors declare no competing interests.

Open practice statement The data from all experiments are available on the Open Science Framework (https://osf.io/pg2x8/). None of the experiments described were preregistered.

\section{References}

Benedetto, A., \& Morrone, M. C. (2017). Saccadic suppression is embedded within extended oscillatory modulation of sensitivity. The Journal of Neuroscience 37, 3661-3670. doi: https://doi.org/10. 1523/jneurosci.2390-16.2016

Billeke, P., Zamorano, F., Cosmelli, D., \& Aboitiz, F. (2012). Oscillatory brain activity correlates with risk perception and predicts social decisions. Cerebral Cortex, 23, 2872-2883. doi: https://doi.org/10. 1093/cercor/bhs269

Bollimunta, A., Mo, J., Schroeder, C. E., \& Ding, M. (2011). Neuronal mechanisms and attentional modulation of corticothalamic $\alpha$ oscillations. The Journal of Neuroscience 31, 4935-4943. doi: https://doi. org/10.1523/JNEUROSCI.5580-10.2011

Cavanagh, J. F., \& Frank, M. J. (2014). Frontal theta as a mechanism for cognitive control. Trends in Cognitive Sciences 18, 414-421. doi: https://doi.org/10.1016/j.tics.2014.04.012

Cavanagh, J. F., Frank, M. J., Klein, T. J., \& Allen, J. J. B. (2010). Frontal theta links prediction errors to behavioral adaptation in reinforcement learning. Neuroimage, 49, 3198-3209. doi: https://doi.org/10. 1016/j.neuroimage.2009.11.080

Chauvigné, L. A. S., Walton, A., Richardson, M. J., \& Brown, S. (2019). Multi-person and multisensory synchronization during group dancing. Human Movement Science, 63, 199-208. doi: https://doi.org/10. 1016/j.humov.2018.12.005

Cheng, X., Li, X., \& Hu, Y. (2015). Synchronous brain activity during cooperative exchange depends on gender of partner: A fNIRS-based hyperscanning study. Human Brain Mapping, 36, 2039-2048. doi: https://doi.org/10.1002/hbm.22754

Cui, X., Bryant, D. M., \& Reiss, A. L. (2012). NIRS-based hyperscanning reveals increased interpersonal coherence in superior frontal cortex during cooperation. Neuroimage, 59, 2430-2437. doi: https://doi.org/10.1016/j.neuroimage.2011.09.003

Cuijpers, L. S., Den Hartigh, R. J. R., Zaal, F. T. J. M., \& de Poel, H. J. (2019). Rowing together: Interpersonal coordination dynamics with and without mechanical coupling. Human Movement Science, 64, 38-46. doi: https://doi.org/10.1016/j.humov.2018.12.008

de Cheveigné, A., \& Arzounian, D. (2018). Robust detrending, rereferencing, outlier detection, and inpainting for multichannel data. Neuroimage, 172, 903-912. doi: https://doi.org/10.1016/j. neuroimage.2018.01.035

Fairhurst, M. T., Janata, P., \& Keller, P. E. (2014). Leading the follower: An fMRI investigation of dynamic cooperativity and leaderfollower strategies in synchronization with an adaptive virtual partner. Neuroimage, 84, 688-697. doi: https://doi.org/10.1016/j. neuroimage.2013.09.027

Fiebelkorn, I. C., \& Kastner, S. (2019). A rhythmic theory of attention. Trends in Cognitive Sciences 23, 87-101. doi: https://doi.org/10. 1016/j.tics.2018.11.009

Fiebelkorn, I. C., Saalmann, Yuri B., \& Kastner, S. (2013). Rhythmic sampling within and between objects despite sustained attention at a cued location. Current Biology, 23, 2553-2558. doi: https://doi. org/10.1016/j.cub.2013.10.063

Genovese, C. R., Lazar, N. A., \& Nichols, T. (2002). Thresholding of statistical maps in functional neuroimaging using the false discovery rate. Neuroimage, 15, 870-878. doi: https://doi.org/10.1006/nimg. 2001.1037

Ho, H. T., Leung, J., Burr, D. C., Alais, D., \& Morrone, M. C. (2017). Auditory sensitivity and decision criteria oscillate at different frequencies separately for the two ears. Current Biology, 27, 36433649.e3643. doi: https://doi.org/10.1016/j.cub.2017.10.017

Ho, H. T., Burr, D. C., Alais, D., \& Morrone, M. C. (2019). Auditory perceptual history is propagated through alpha oscillations. Current Biology. doi: https://doi.org/10.1016/j.cub.2019.10.041

Hu, Y., Pan, Y., Shi, X., Cai, Q., Li, X., \& Cheng, X. (2018). Inter-brain synchrony and cooperation context in interactive decision making. Biological Psychology, 133, 54-62. doi: https://doi.org/10.1016/j. biopsycho.2017.12.005

Huang, Y., Chen, L., \& Luo, H. (2015). Behavioral oscillation in priming: Competing perceptual predictions conveyed in alternating thetaband rhythms. The Journal of Neuroscience 35, 2830-2837. doi: https://doi.org/10.1523/jneurosci.4294-14.2015

Jiang, J., Chen, C., Dai, B., Shi, G., Ding, G., Liu, L., \& Lu, C. (2015). Leader emergence through interpersonal neural synchronization. Proceedings of the National Academy of Sciences, 112, 42744279. doi: https://doi.org/10.1073/pnas.1422930112

Kawasaki, M., Yamada, Y., Ushiku, Y., Miyauchi, E., \& Yamaguchi, Y. (2013). Inter-brain synchronization during coordination of speech rhythm in human-to-human social interaction. Scientific Reports, 3, 1692. doi: https://doi.org/10.1038/srep01692

Konvalinka, I., Vuust, P., Roepstorff, A., \& Frith, C. D. (2010). Follow you, follow me: Continuous mutual prediction and adaptation in joint tapping. Quarterly Journal of Experimental Psychology, 63, 2220-2230. doi: https://doi.org/10.1080/17470218.2010.497843

Konvalinka, I., Bauer, M., Stahlhut, C., Hansen, L. K., Roepstorff, A., \& Frith, C. D. (2014). Frontal alpha oscillations distinguish leaders from followers: Multivariate decoding of mutually interacting brains. Neuroimage, 94, 79-88. doi: https://doi.org/10.1016/j. neuroimage.2014.03.003

Landau, A. N. (2018). Neuroscience: A mechanism for rhythmic sampling in vision. Current Biology, 28, R830-R832. doi: https://doi. org/10.1016/j.cub.2018.05.081

Landau, A. N., \& Fries, P. (2012). Attention samples stimuli rhythmically. Current Biology, 22, 1000-1004. doi: https://doi.org/10.1016/j.cub. 2012.03.054

Liu, D., Liu, S., Liu, X., Zhang, C., Li, A., Jin, C., ... Zhang, X. (2018). Interactive brain activity: Review and progress on EEG-based hyperscanning in social interactions. Frontiers in psychology, 9. doi: https://doi.org/10.3389/fpsyg.2018.01862

Re, D., Inbar, M., Richter, C. G., \& Landau, A. N. (2019). Feature-based attention samples stimuli rhythmically. Current Biology, 29, 693699.e694. doi: https://doi.org/10.1016/j.cub.2019.01.010

Richardson, M., Garcia, R., Frank, T., Gregor, M., \& Marsh, K. (2012). Measuring group synchrony: a cluster-phase method for analyzing multivariate movement time-series. Frontiers in Physiology, 3. doi: https://doi.org/10.3389/fphys.2012.00405

Rohenkohl, G., \& Nobre, A. C. (2011). Alpha oscillations related to anticipatory attention follow temporal expectations. The Journal of Neuroscience 31, 14076-14084. doi: https://doi.org/10.1523/ jneurosci.3387-11.2011

Sänger, J., Müller, V., \& Lindenberger, U. (2012). Intra- and interbrain synchronization and network properties when playing guitar in duets. Frontiers in Human Neuroscience, 6. doi: https://doi.org/10. 3389/fnhum.2012.00312

Silva, P., Chung, D., Carvalho, T., Cardoso, T., Davids, K., Araújo, D., \& Garganta, J. (2016). Practice effects on intra-team synergies in football teams. Human Movement Science, 46, 39-51. doi: https://doi. org/10.1016/j.humov.2015.11.017

Song, K., Meng, M., Chen, L., Zhou, K., \& Luo, H. (2014). Behavioral oscillations in attention: Rhythmic $\alpha$ pulses mediated through $\theta$ 
band. The Journal of Neuroscience 34, 4837-4844. doi: https://doi. org/10.1523/jneurosci.4856-13.2014

Tomassini, A., Spinelli, D., Jacono, M., Sandini, G., \& Morrone, M. C. (2015). Rhythmic oscillations of visual contrast sensitivity synchronized with action. The Journal of Neuroscience 35, 7019-7029. doi: https://doi.org/10.1523/jneurosci.4568-14.2015

VanRullen, R. (2013). Visual attention: A rhythmic process? Current Biology, 23, R1110-R1112. doi: https://doi.org/10.1016/j.cub.2013. 11.006
VanRullen, R. (2016). Perceptual cycles. Trends in Cognitive Sciences 20, 723-735. doi: https://doi.org/10.1016/j.tics.2016.07.006

Publisher's note Springer Nature remains neutral with regard to jurisdictional claims in published maps and institutional affiliations. 